\section{Re: The adult hip joint in Saudi Arabia}

To the Editor: The hip joint presents some of the most fascinating problems in the field of orthopedic surgery. Injuries and diseases of the adult hip are important as they may cause prolonged suffering and serious disability. A review of the Saudi literature to explore the pattern of traumatic and non-traumatic disorders affecting the adult hip joint in Saudis has revealed many interesting observations that raise unanswered questions.

First, osteopenia and osteoporosis were reported to be prevalent $(70 \%)$ in Saudi females above the age of 50 years, ${ }^{1}$ yet osteoporosisrelated hip fractures are uncommon compared to many other populations. The incidence of hip fractures per 100000 for male and female patients over the age of 50 years was 71 and 100 , respectively, with a female: male ratio of 1.4. This is much less than the incidence of hip fractures in other Western populations. ${ }^{2}$

Secondly, despite the high prevalence of knee osteoarthritis (OA), hip $O A$ is very uncommon in Saudi Arabia, reaching $0.04 \%$ with $80: 1$ ratio of knee to hip OA., ${ }^{5,6}$ This is very low compared to other populations. Developmental dysplasia of the hip is relatively common in Saudi Arabia, ${ }^{7.9}$ and acetabular dysplasia in adults has not been studied. Both conditions are well known risk factors for hip OA. Furthermore, the main indication for hip arthroplasty among Saudis is avascular necrosis of the femoral head secondary to sickle cell disease, ${ }^{10.11}$ and not hip OA in elderly patients as is the case in other populations. This type of surgery is done mainly for young adults with sickle cell disease and carries a higher risk of complication.
Thirdly, two studies have shown a unique difference in hip and femoral geometry; both rotational ${ }^{12}$ and axial ${ }^{13}$ alignments, in Saudi arthritic knees. Whether this is due to a developmental factor or an adaptive reparative change secondary to $O A$ is unknown. A metabolic element was also presumed to be contributing for this femoral and hip malalignment.

Fourthly, one of the authors has shown that hip joints in Saudis have a significantly increased range of motion in abduction and external rotation compared with western populations. ${ }^{14}$ This is considered to be related to local habitual crosslegged sitting positions among Saudis. We think this cultural habit has a favorable effect on the hip joint mechanics, nutrition, development and containment.

Whether all these features of the "Saudi hip" are due to special geometric morphologic factors, kinematic elements, or racial genetic and metabolic variables is a matter of speculation. Further studies are needed to elaborate these special "unique" features of adult Saudi hip joints.

\section{Mohammed Moussa, Abdulla \\ Alumran \\ Correspondence and reprint requests: \\ Dr. Mohammed Moussa \\ King Fahd University Hospital, \\ Al-Khobar PO Box 2208 \\ 31952- Saudi Arabia \\ aboalimoussa@yahoo.com \\ Tel. : 0020127440264}

\section{REFERENCES}

1. El-Desouki MI.. Osteoporosis in postmenopausal Saudi women using dual $\mathrm{x}$-ray bone densitometry. Saudi Med J. 2003; 24(9):953-6.

2 Al-Nuaim AR, Kremli M, al-Nuaim M, Sandkgi S. Incidence of proximal femur fracture in an urbanized community in Saudi Arabia. Calcif Tissue Int 1995; 56(6):536-8.

3. Ahlberg $A$, Moussa $M$. The anatomical pattern of gonarthrosis in Saudi Arabia. Int Orthop. 1989;13(3):177-8.

4. Al-Arfaj AS, Alballa SR, AI-Saleh SS, AI-Dalaan AM, Bahabry SA, Mousa MA, Al-Sekeit MA. Knee osteoarthritis in Al-Qaseem, Saudi Arabia. Saudi Med J. 2003; 24(3):291-3.
5. Agunwa WC. Low incidence of osteoarthrosis of the hips in contrast with osteoarthrosis of the knees among rural Saudis: why? Ann Rheum Dis. 1989; 48(4):351-2.

6. Ahlberg A, Linder B, Binhemd TA.Osteoarthritis of the hip and knee in Saudi Arabia. Int Orthop. 1990; 14(1):29-30.

7. Al-Umran K. Neonatal hip instability in Saudi Arabia: Results and cost effectiveness. Annals of Saudi Medicine. 1994; Vol 14 (1):16-18.

8. Mirdad $T$. Incidence and pattern of congenital dislocation of the hip in Aseer region of Saudi Arabia. West Afr J Med. 2002; 21(3):218-22.

9. Moussa M, Al-Othman A. Bilateral developmental dysplasia of the hip: asymmetric outcome in the older child. Clin Orthop. 2001;(392):358-65.

10. Sanjay BK, Moreau PG. Bipolar hip replacement in sickle cell disease. Int Orthop. 1996;20(4):222-6. 11. Ilyas I, Moreau P. Simultaneous bilateral total hip arthroplasty in sickle cell disease. J Arthroplasty. 2002;17(4):441-5.

12 Moussa $M$. Rotational malalignment and femoral torsion in osteoarthritic knees with patellofemoral joint involvement. A CT scan study. Clin Orthop. 1994;(304):176-83.

13. Cooke TD, Harrison L, Khan B, Scudamore A Chaudhary MA. Analysis of limb alignment in the pathogenesis of osteoarthritis: a comparison of Saudi Arabian and Canadian cases. Rheumatol Int 2002; 22(4):160-4.

14. Ahlberg A, Moussa M, Al-Nahdi M. On geographical variations in the normal range of joint motion. Clin Orthop. 1988;(234):229-31.

\section{Re: Epidermoid cyst of the cecum in an elderly man}

To the Editor: Let me offer an addendum to the article entitled "Epidermoid cyst of the cecum in an elderly man" by Ali Uzunlar and Yasar Burke, Ann Saudi Med 2006;26(6):477-479.

There has been another paper on this topic published in 2003 that was not mentioned by the authors in their review, namely: Reyes $\mathrm{CV}$ et al Epithelial inclusion cyst of the cecum, Practical Gastroenterology 2003;27(9):52-58.

Briefly, a 66-year old man who presented with acute right lower quadrant abdominal pain, which was interpreted atypical for acute appendicitis. A subserosal/muscular cystic mass in the cecum without luminal communication but accompanied by tiny pockets of free air and acute inflammatory changes 
in the right colonic gutter was noted on computed tomographic scan with contrast. On exploration, no obvious perforation of any viscus but a tracking of purulent material along the right pericecal gutter was found. With a palpation finding of a soft mass in the cecal wall and low suspicion for malignancy, only a short segment ileocecectomy was performed. The patient had uneventful postoperative follow up.

The lesion microscopically was lined by stratified squamous epithelium, contained ample keratinous material, and associated with a granular cell tumor wall in the subepithelial soft tissue and surrounding smooth muscle. Special studies (periodic acid-Schiff and immunostaining with neuron specific enolase, S-100, lyzosymes, desmin and vimentin) affirmed a reactive histiocytic nature of the granular cells.

The paper was also well illustrated with four figures, including a CT scan with contrast, gross photograph of the surgical specimen, microscopic findings of the keratinized squamous epithelial lining, and an incidental granular cell tumor was found in the wall of the cyst.

A literature review was also presented.

Cesar V. Reyes

Staff Physician

Morris Hospital

150 West High Street

Morris, IL 60450

USA

creyes@morrishospital.org

\section{Simultaneous bilateral spontaneous pneumo- thorax: a case report}

To the Editor: Pneumothorax is the presence of air in the pleural space with secondary lung collapse. Simultaneous bilateral spontaneous pneumothorax (SBSP), defined as the concurrent occurence of pneumothorax in both hemithoraces is a rare clinical condition in the literature, mainly seen in patients with underlying lung disease. ${ }^{1}$ The incidence of spontaneous pneumothorax is 9 per 100000 and 1.3 percent of these cases are bilateral and simultaneous. ${ }^{2}$ We describe a young patient with simultaneous bilateral spontaneous pneumothorax.

A 23-year-old patient was admitted to our emergency service 1 hour after the sudden onset of dyspnea and chest pain. There was no history of trauma to the chest. He denied any history of smoking. Physical examination showed that the patient was a tall, thin man. He was tachycardic and normotensive. He was in apparent distress with a respiratory rate of 22 breaths/minute. The examination of the chest revealed that breath sounds were decreased bilaterally. The percussion note was resonant in all areas of the chest. The trachea was not displaced. Laboratory findings were normal.

The chest radiograph confirmed total pneumothorax on the right side and partial pneumothorax on the left side (Figure 1). Simultaneous tube thoracostomy was carried out on both the right and left hemithorax. Both lungs were soon fully reexpanded. A computed tomography scan was normal. No underlying emphysematous change or bullous disease was identified. We decided to operate on the left side because the air leak was continuous. But the patient did not accept the operation. As the air leak stopped on the right side on the fifth day and on the left side on the seventh day, the thoracic tubes were removed. To prevent any recurrence, bilateral chemical pleurodesis with tetracycline was performed. The patient was discharged on day 9 and was asymptomatic at 5 months follow- up.

Spontaneous pneumothorax is more frequent in young, tall and thin males, and more common in smokers. The most common cause of spontaneous pneumothorax is the rupture of small subpleural bleps. Simultaneous bilateral spontaneous pneumothorax is a rare clinical event. There are isolated reports of bilateral pneumothorax secondary to trauma, malignancy, high-pressure ventilation and catheterization.

Most case reports in the literature were associated with malignancy. ${ }^{1,3}$ A few cases of simultaneous bilateral primary spontaneous pneumothorax have been reported in the literature, as in our case.

The clinical presentation of SBSP can be variable. Patients have presented with clinical signs and symptoms ranging from cardiopulmonary failure to as little as mild dyspnea. ${ }^{4}$ Symptoms may be equal bilaterally. Spontaneous pneumothorax is generally diagnosed by the clinical features. The diagnosis should be based on radiological findings. The chest radiograph constitutes the basis of radiological examination. CT of the chest is indicated to detect underlying causes of spontaneous pneumothorax. ${ }^{3,4}$ Our patient presented with typical signs and symptoms of pneumothorax and the CT scan was normal.

The treatment options for pneumothorax range from observation to thoracotomy. These treatment options include needle aspiration, percutaneous catheter drainage, tube thoracostomy with or without chemical pleurodesis, and videoassisted thoracic surgery. There are some controversies about the treatment of simultaneous bilateral spontaneous pneumothorax. Initially, chest drainage is essential in the treatment. ${ }^{3.5}$

The main goals of pneumotho- 